\title{
Chronic cutaneous lupus erythematosus
}

INSERM

\section{Source}

INSERM. (1999). Orphanet: an online rare disease and orphan drug data base. Chronic cutaneous lupus erythematosus. ORPHA:163531

Chronic cutaneous lupus erythematosus (CCLE) is a form of cutaneous lupus erythematosus (CLE; see this term) that includes five different forms: discoid lupus erythematosus (DLE), chilblain lupus, hypertrophic or verrucous lupus erythematosus, lupus erythematosus tumidus, and lupus erythematosus panniculitis (see these terms). 